\title{
DLC1 suppresses distant dissemination of human hepatocellular carcinoma cells in nude mice through reduction of RhoA GTPase activity, actin cytoskeletal disruption and down-regulation of genes involved in metastasis
}

\author{
XIAOLING ZHOU $^{1}$, DRAZEN B. ZIMONJIC ${ }^{1}$, SANG-WON PARK ${ }^{1,2}$, XU-YU YANG $^{1}$, \\ MARIAN E. DURKIN ${ }^{1}$ and NICHOLAS C. POPESCU ${ }^{1}$ \\ ${ }^{1}$ Laboratory of Experimental Carcinogenesis, Center for Cancer Research, \\ National Cancer Institute, Bethesda, MD 20892, USA
}

Received January 7, 2008; Accepted February 21, 2008

\begin{abstract}
The process of cell dissemination from the primary tumors to distant sites is the most harmful event during cancer progression, and the leading cause of cancer death. We have previously demonstrated that restoration of DLC1 tumor suppressor gene expression in the DLC1-negative Focus and $7703 \mathrm{~K}$ human hepatocellular carcinoma (HCC) cell lines induced caspase- 3 mediated apoptosis, reduced cell growth in vitro and tumorigenicity in vivo and diminished the ability to migrate through Matrigel, a property suggestive of metastatic potential in vivo. We now show that subcutaneous tumors developing after inoculation of Focus and 7703K cells into nude mice disseminate cells to liver and lung, and this process is markedly suppressed by restoration of DLC1 expression. Inhibition of tumor cell dissemination was associated with lower levels of RhoA activity, an increase in rounded cells and a reduction in actin stress fibers and focal adhesion molecules that are of critical importance in cancer cell invasion and metastasis. In addition, DLC1 down-regulated the expression of osteopontin and matrix metalloproteinase-9, which are highly up-regulated in most primary HCC with associated metastases. These observations implicate the DLC1 gene in suppression of HCC cell dissemination and identify novel cellular and genetic alterations that contribute to prevention of metastasis, a life-threatening event in cancer progression.
\end{abstract}

Correspondence to: Dr Nicholas C. Popescu, Laboratory of Experimental Carcinogenesis, National Cancer Institute, 37 Covent Drive, MSC 4262, Bethesda, MD 20892, USA

E-mail: popescun@mail.nih.gov

Present address: ${ }^{2}$ Department of Biology, Beckman Research Institute, City of Hope National Medical Center, Duarte, CA, USA

Key words: DLC1, tumor suppressor gene, Rho GTPase activating protein, liver cancer, metastasis, osteopontin, metalloproteinase- 9

\section{Introduction}

The process of tumor cell dissemination from locally growing primary tumors to anatomically distant sites is the most deleterious event during cancer progression and the leading cause of cancer mortality. Primary tumors account for only about $10 \%$ of deaths from cancer, while metastatic tumors developing at secondary organs are responsible for the remainder of cancer casualties (1). Metastatic disease is a complex process involving various cellular and genetic alterations. Despite intensive efforts, the mechanisms of cancer cell dissemination remain elusive and only a handful of genes have been implicated in this process (1-3).

DLC1 (deleted in liver cancer) was first identified as a gene underepresented in human primary hepatocellular carcinomas (HCC) and subsequently found to be frequently inactivated or down-regulated in a variety of solid tumors and hematological malignancies (4, reviewed in ref. 5). The DLC1 gene encodes a 1091-amino acid polypeptide that contains a Rho GTPase activating protein (Rho GAP) domain, a conserved domain present in proteins that act as negative regulators of the Rho family of small GTPases (4). DLC1 functions as a tumor suppressor gene in breast, lung, ovarian, nasopharyngeal, esophageal, cervical and liver cancer, and it was also found to suppress metastasis of human breast cancer cells in nude mouse model systems $(5,6)$. We have previously demonstrated that restoration of DLC1 expression in two HCC cell lines that lack endogenous DLC1 expression, Focus and $7703 \mathrm{~K}$, inhibited cell growth in vitro, induced apoptosis, and reduced the ability of cells to form tumors in athymic nude mice. Since DLC1 also inhibited the ability of the cells to migrate through Matrigel, this raised the possibility that these cells may provide a model for studying HCC invasion and metastases in nude mice (7).

We now examined the ability of Focus and $7003 \mathrm{~K}$ HCC cells to spread to secondary sites and assessed the effect of DLC1 on this process. Our observations show that tumor cells from both HCC lines disseminate to the liver and lung and their migration is suppressed in DLC1stably transfected cells. The suppressive effect of DLC1 
on tumor cell dissemination was accompanied by activation of RhoA GTPase, alterations in cytoskeleton organization and cell adhesion as well as in down-regulation of two genes, osteopontin (OPN) and metalloproteinase-9 (MMP-9), that are considered to be critical in metastatic process of HCCs.

\section{Materials and methods}

Cell lines and cell culture. Focus and 7703K cells stably transfected with the pcDNA3.1 vector (Invitrogen, Carlsbad, CA, USA) containing cDNA encoding the human DLC1 open reading frame and with the empty vector have been described (7). The cells were cultured in a 1:1 mixture of Dulbecco's modified Eagle's medium and F-12 (DMEM-F12) supplemented with $10 \%$ fetal bovine serum, $2 \mathrm{mM}$ glutamine, penicillin $(100 \mathrm{U} / \mathrm{ml})$, streptomycin $(100 \mu \mathrm{g} / \mathrm{ml})$ and $250 \mu \mathrm{g} / \mathrm{ml}$ G418 (Invitrogen).

Detection of in vivo cell invasion. Cells stably transfected with DLC1 (Focus/DLC1 and 7703K/DLC1) and vector only (Focus/Vector and 7703K/Vector) were cultured in medium containing G418 and harvested. After cell viability was determined, $2 \times 10^{6}$ viable cells were injected subcutaneously at the proximal dorsal midline of 6-week-old male athymic nu/mu mice (Harlan). The same amount of phosphate-buffered saline (PBS) solution was injected in mock-inoculated mice. Ten mice were used for each experiment group. Tumor development was monitored and the size of the tumors was measured in 2 dimensions twice a week. When tumor size reached $6 \times 8 \mathrm{~mm}$, the mice were sacrificed and tumor, lung and liver tissues were excised. Genomic DNAs were extracted with DNeasy tissue kit (Qiagen, Inc., Valencia, CA) for PCR analysis. The liver and lung tissues were fixed in $10 \%$ buffered formalin overnight at room temperature for histological preparations.

Tumor cell dissemination was measured by a quantitative PCR (qPCR) assay using genomic DNA from mouse tissues and human Alu sequence-specific primers: sense, 5'-CAC CTG TAA TCC CAG CAC TTT-3'; anti-sense, 5'-CCC AGG CTG GAG TGC AGT-3'. Mouse GAPDH primers were used as an internal control (Applied Biosystems, Foster City, CA). Amplifications were performed with a SYBR-Green PCR kit (Invitrogen) and the ABI PRISM ${ }^{\circledR} 7900$ Sequence Detection System (Applied Biosystems) under Universal Cycling Conditions. The $2^{-\Delta \Delta \mathrm{Ct}}$ method was used to calculate the relative fold difference of human Alu sequence in all samples compared to the normal samples from mock inoculated mice.

Transient adenovirus DLC-1-mediated expression. Adenoviruses expressing human DLC1 were prepared with the ViraPower Adenoviral Expression System (Invitrogen) using the Gateway method. Briefly, cDNA encoding the DLC1 open reading frame was subcloned into the $\mathrm{pENTR/D-TOPO}$ vector, and the insert was transferred to the pAd/CMV/V5DEST vector. The pAd-DLC1 vector and the pAd-LacZ control vector were transiently transfected into 293A cells for production of adenovirus. The Ad-DLC1 and Ad-LacZ viruses were purified and stored in aliquots at $-80^{\circ} \mathrm{C}$. The viral titer (plaque-forming unit) was determined using 293A cells according to the manufacturer's instructions.

Focus and $7703 \mathrm{~K}$ cells were seeded at $2 \times 10^{6}$ cells/ $100-\mathrm{mm}$ dish $24 \mathrm{~h}$ prior to adenoviral infection. Ad-DLC1 and Ad-LacZ were added at MOIs (multiplicity of infection) 100 in $8 \mathrm{ml}$ of medium. After 24-h infection, the Focus/ Ad-DLC1, Focus/Ad-LacZ, 7703K/Ad-DLC-1 and 7703K/ Ad-LacZ cells were cultured in complete fresh medium for another $24 \mathrm{~h}$ before further use.

Rho activation assay. The relative RhoA activities in Focus/ Ad-DLC1, Focus/Ad-LacZ, 7703K/Ad-DLC1 and 7703K/ Ad-LacZ cells were measured with an ELISA-based RhoA activation assay kit (G-LISA, Cytoskeleton, Inc., Denver, $\mathrm{CO}$ ) according to the manufacturer's instructions. After serum-starvation and lysophosphatidic acid (LPA) stimulation, cells were lysed and the protein concentration was determined according to the manufacturer's protocol. The lysates were incubated in micro-wells coated with the Rhotekin Rho-binding domain, and active RhoA was measured using indirect immunodetection followed by a colorimetric reaction measured by absorbance at $490 \mathrm{~nm}$.

Visualization of actin filaments and focal adhesion. The procedures for immunofluorescent microscopy detection of actin fibers with phalloidin and of focal adhesions with anti-vinculin antibodies were previously described in detail (8).

Real-time quantitative RT-PCR analysis. Total RNA was isolated from Focus and $7703 \mathrm{~K}$ cells and transcribed into cDNA. The ABI PRISM 7900HT Sequence Detection System (PE Applied Biosystems) was used for real-time cDNA quantitation of DLC1, OPN and MMP-9 mRNA. The primer sets and probes were also purchased from Applied Biosystems. Human GAPDH was used as an endogenous control (TaqMan human GAPDH Control Reagents kit, Applied Biosystems). The $2^{-\Delta \Delta C t}$ method was used to calculate the relative fold difference of DLC1 and OPN mRNA expressions in all samples.

Western blot analysis. Proteins were isolated with IP buffer from Focus and 7703K cultures, and total protein concentration was determined using the Bio-Rad (Hercules, CA) assay. Total protein $(50 \mu \mathrm{g})$ were loaded per well. Blotted membranes were incubated with primary antibody against OPN (Chemicon International, Inc., Temecula, CA), ROCK-I, ROCK-II or DLC1 (BD Biosciences, San Jose, CA).

Statistics. All data are representative of three independent experiments. The significance of differences between means was determined by Student's t-test. A P-value of $<0.05$ was considered statistically significant.

\section{Results}

DLC1 effect on distant dissemination of HCC cells. We previously showed that Focus and $7703 \mathrm{~K}$ cell lines formed tumors in nude mice, and that tumor size was reduced in cells transfected with DLC1 (7). Since both cell lines displayed an 


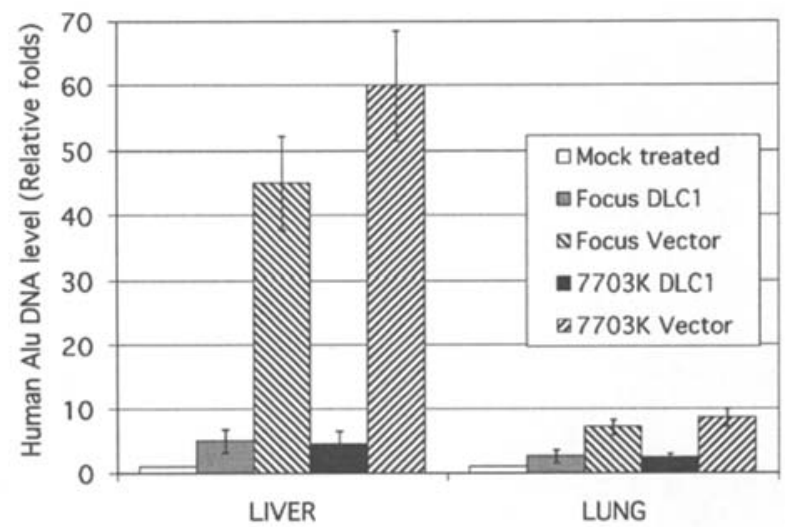

Figure 1. Detection of human tumor cells dissemination in liver and lung of athymic mice. The levels of human Alu DNA, representing dissemination of subcutaneously injected HCC cells, were quantitated by real-time PCR. Three- to 13-fold more human Alu DNA were detected in the lungs and livers of mice injected with $7703 \mathrm{~K} /$ Vector and Focus/Vector cells than in tissues of mice injected with 7703K/DLC1 and Focus/DLC1 cells. There was no meaningful level of human Alu DNA in lungs and livers of mock injected mice.

ability to migrate through Matrigel, an in vitro manifestation of cell invasiveness, we examined whether the invasive property was reflected in vivo and whether DLC1 expression had an impact on this process. Focus and $7703 \mathrm{~K}$ cells stably transfected with DLC1 or the empty vector were injected subcutaneously into athymic nude mice. Consistent with our previous observations, within one month after inoculation the two HCC cell lines lacking DLC1 produced tumors that were more numerous and considerably larger than DLC1expressing cells (7). For a fair comparison of the capacity of cells to disseminate to distant organs, the mice inoculated with DLC1-positive cells were kept, monitored regularly and sacrificed when the tumors reached the same size as those produced by DLC1-negative cells, prior to the onset of morbidity due to excessive tumor size. To detect dissemination of the tumor cells to liver and lung, a highly sensitive qPCR assay using human Alu sequences that is increasingly used for detection of metastases, was employed (6,9-11). The Alu primers show high specificity for human DNA, and the assay detects human DNA as low as $500 \mathrm{fg} / 20 \mu \mathrm{l}$ (9). A higher amount of human DNA (6-7 times, $\mathrm{P}<0.05)$ from both of the cell lines transfected with empty vector was detected in the liver than in lung (Fig. 1). Three to 13 times higher levels of human Alu sequences $(\mathrm{P}<0.01)$ were detected in liver and lung tissue of mice bearing subcutaneous tumors that developed after inoculation of $7703 \mathrm{~K}$ and Focus transfected with the empty vector compared to those transfected with DLC1 (Fig. 1). 7703K cells showed more aggressive dissemination than Focus cells whereas in PBS mock inoculated mice only background level of noise was detected (Fig. 1). Microscopic metastases were not observed in histological preparations from either liver or lung at this limited tumor size and time frame.

DLC1 effect on RhoA activity. To determine the effects of restoration of DLC1 expression on cell behavior, we developed an adenoviral expression system to maximize the

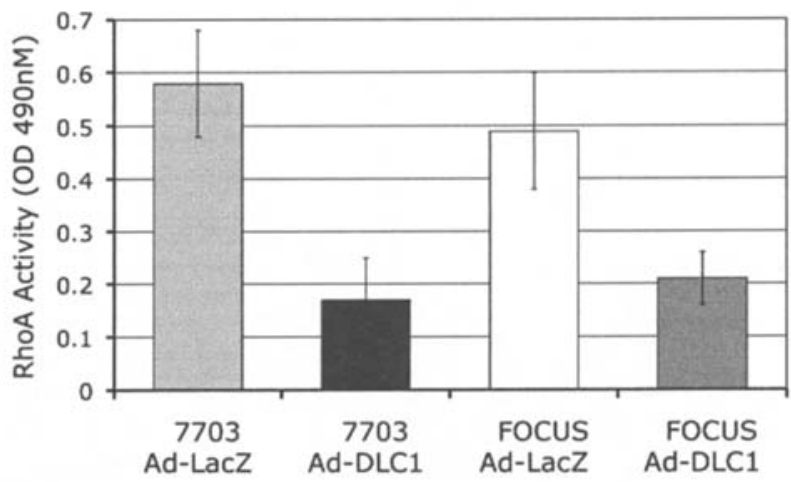

Figure 2. Expression of DLC1 inhibits RhoA activity. RhoA activity was two to three times lower in cells transduced with DLC1 (7703K/Ad-DLC1 and Focus/Ad-DLC1) than in cells transduced with the LacZ control (7703K/Ad-LacZ and Focus/Ad-LacZ). The data shown are the mean levels of RhoA activity \pm SE from three independent experiments.

efficiency of gene transfer and to achieve high levels of transgene expression in a short time. The adenoviral vector bearing the DLC1 cDNA was used for transient transduction of HCC cells, and the DLC1 transduction efficiency was about $100 \%$ at one day after transduction detected by immunofluorescent staining (data not shown). RhoA activity, cellular morphological alterations and the expression of several genes that are known to be implicated in metastatic progression of HCC were examined in the Ad-DLC1 and Ad-LacZ infected Focus and $7703 \mathrm{~K}$ cells.

To determine whether overexpression of DLC1 was accompanied by reduced activation of RhoA, we used an ELISA-based RhoA activation assay. The levels of active, GTP-bound RhoA in Focus/Ad-DLC1 and 7703K/Ad-DLC1 cells were 40-60\% lower than Focus/Ad-LacZ and 7703K/ Ad-LacZ cells ( $\mathrm{P}=0.026$; Fig. 2$)$.

Reorganization of cytoskeletal and adhesion structures. Tumor cell migration and invasion of foreign tissue space is closely associated with morphological and functional reorganization of its cytoskeletal and adhesive structures. To verify that cytoskeleton reorganization signaling pathways are affected by re-expression of DLC1 we examined the distribution of actin filaments and focal adhesions by staining with rhodamine-conjugated phalloidin and indirect immunofluorescence with anti-vinculin antibody, respectively (Fig. 3). In both Focus and $7703 \mathrm{~K}$ cells re-expression of DLC1 caused significant morphologic changes, as demonstrated by decreased cell spreading, an overall decrease in the number of actin bundles and a significant reduction in the number of mature focal cell-substrate contacts. Changes at the level of actin appear to be more pronounced in Focus cells than in the $7703 \mathrm{~K}$ whereas the effect on focal adhesions appears to be equally profound in both types of cells.

DLC1-mediated down-regulation of genes implicated in metastatic HCC. While the DLC1-mediated deregulation of RhoA activity and altered cytoskeletal organization play an important role in the suppression of HCC cell dissemination, we wished to examine the effect of DLC1 on the expression 

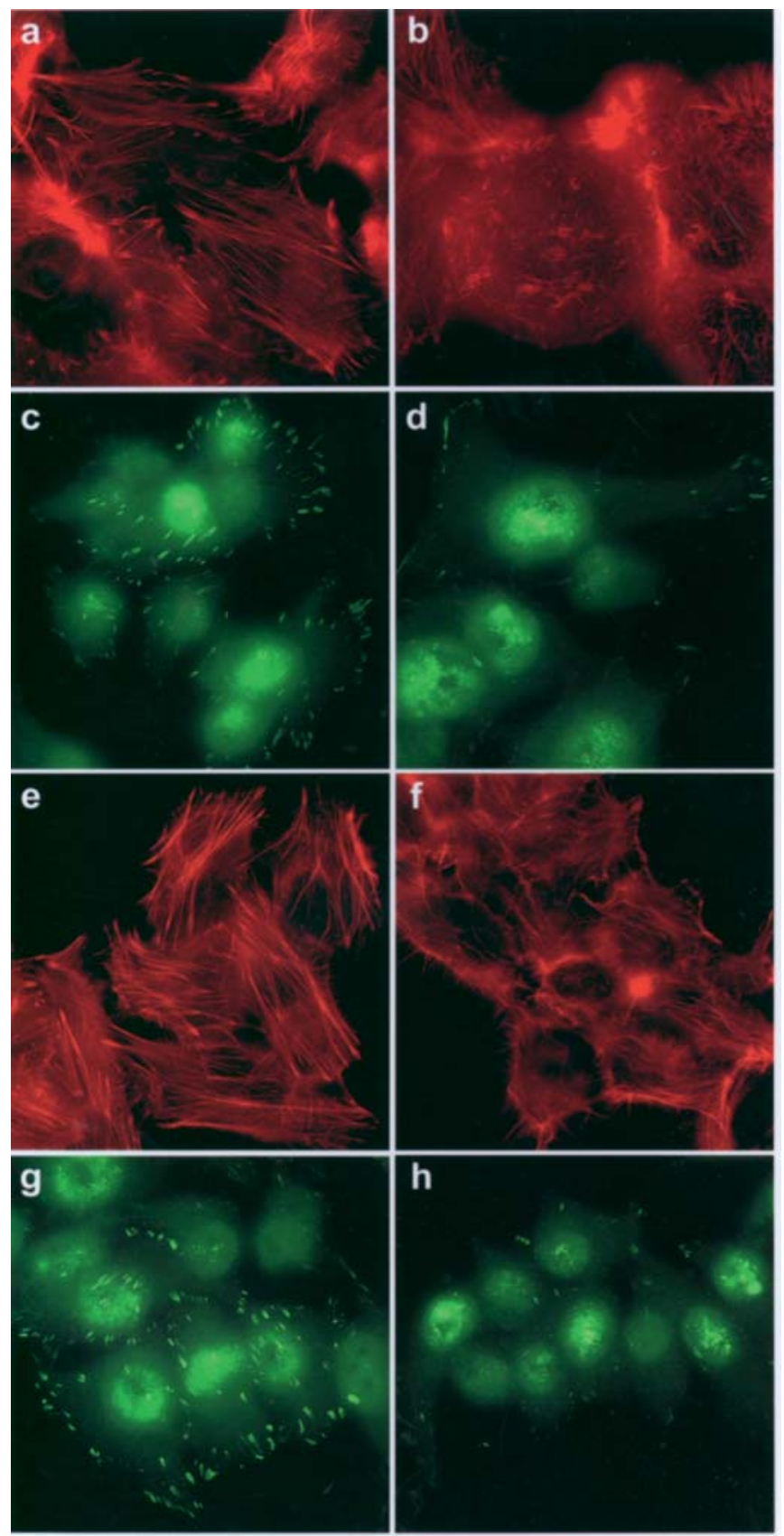

Figure 3. DLC1 expression affects organization and distribution of actin filaments and focal adhesions. Focus (b) and 7703K (f) cells expressing DLC1 exhibit fewer long actin stress fibers compared to their respective DLC1-negative controls (a and e). Similarly, DLC1-positive cells from both Focus (d) and 7703K (h) show a reduced number of vinculin-positive focal adhesion-like structures compared to their respective DLC1-negative controls (c and g).

of other genes known to be involved in metastasis. Since OPN is considered to be a key signature gene in HCC metastasis we sought to determine whether there was a link between DLC1 expression and OPN (12). By real-time RTPCR we detected a 2- to 5-fold decrease of OPN expression $(\mathrm{P}<0.05)$ in DLC1-transduced $7703 \mathrm{~K}$ and Focus cells compared to controls (Fig. 4A). This shows that restoration of DLC1 expression reduced the level of OPN mRNA rather rapidly. To see whether a reduction of OPN expression was manifested at the protein level we examined Focus and $7703 \mathrm{~K}$ cells stably transfected with DLC1 or empty vector
A
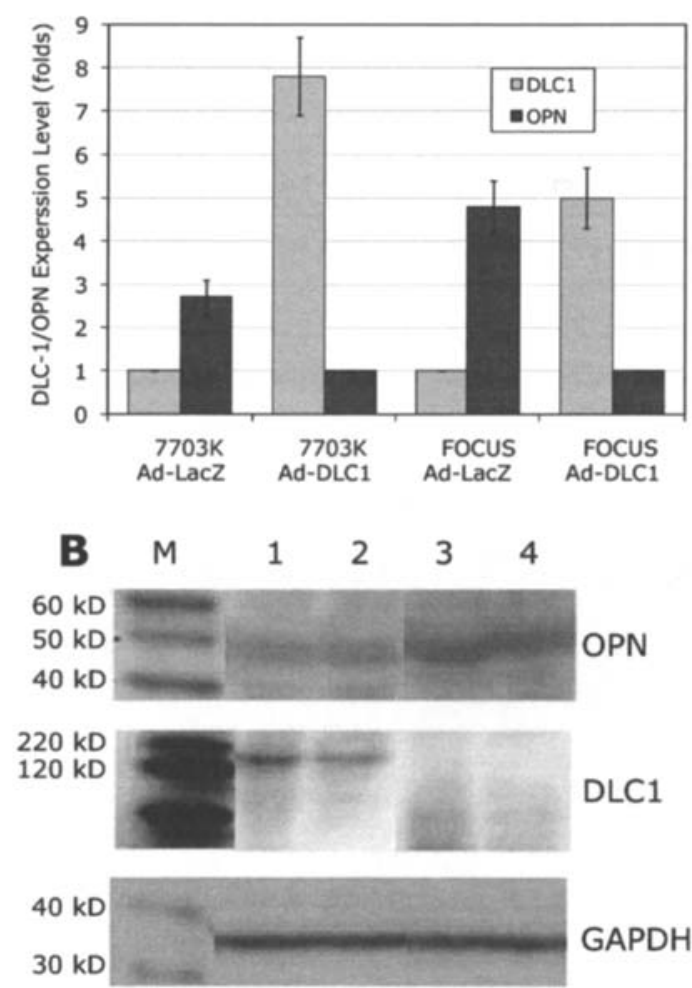

Figure 4. Reduced OPN expression in DLC1-transfected cells. (A) The levels of DLC1 and OPN mRNA in cells transiently transfected with Ad/DLC1 or the control vector were measured by real-time RT-PCR. A significant reduction of OPN gene expression was detected in Focus/ DLC1 and 7703K/DLC1 cells compared to Focus/vector and 7703K/vector cells. (B) Western blotting demonstrated that OPN protein levels were lower in Focus/DLC1 and 7703K/DLC1 stably transfected cells than in control cells. M, molecular weight markers; lane 1, 7703K/DLC1; lane 2, Focus/DLC1; lane 3, 7703K/vector; and lane 4, Focus/vector. Blots were stained with antibodies against OPN (top panel), DLC1 (center panel) and GAPDH (bottom panel).

by Western blotting. The abundance of OPN protein was significantly lower in DLC1 trasfected cells than in control cells (Fig. 4B). In addition to OPN, matrix metalloproteinase-9 (MMP-9) is also up-regulated in metastatic HCC (12) and a recent study demonstrated that cleavage of OPN by MMP-9 might promote HCC metastasis (13). Based on these data we compared the expression of both genes. Real-time PCR analysis showed OPN and MMP-9 mRNA levels and found that were concordantly reduced by 4 - to 5 -fold $(\mathrm{P}<0.05)$ in Focus and $7703 \mathrm{~K}$ cells transduced with the Ad-DLC1 virus compared to Ad-LacZ infected cells (Fig. 5A). Recently, Rho-associated serine-threonine protein kinase (ROCK) was also found to be recurrently overexpressed in primary metastatic HCC and HCC cell lines (14). However, Western blot analysis showed that the abundance of ROCK-I and ROCK-II proteins was seemingly equal in DLC1-positive and negative control cell lines (Fig. 5B).

\section{Discussion}

Migration of primary tumor cells to secondary anatomical sites, also known as 'invasion-metastasis cascade' requires a 
A

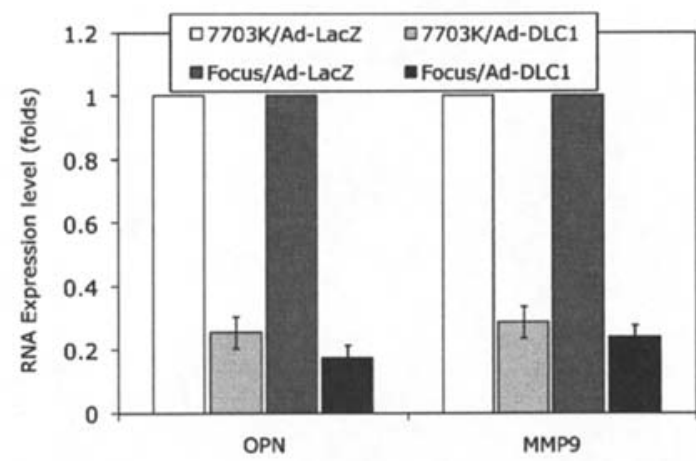

B

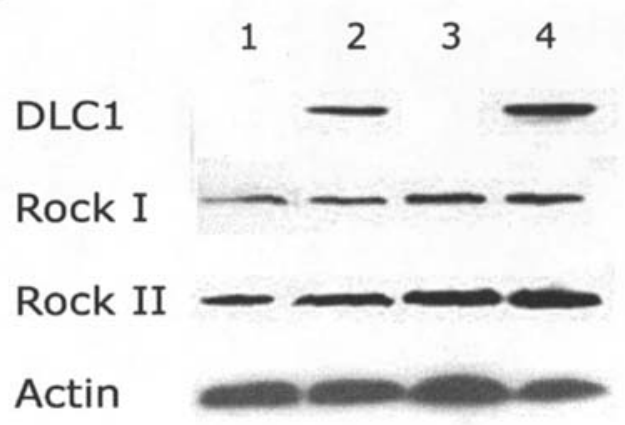

Figure 5. Comparative analysis of DLC1, OPN, MMP-9 and ROCK in DLC1 positive and negative HCC cells. (A) Down-regulation of OPN and MMP-9 expression in Focus/Ad-DLC1 and 7703K/Ad-DLC1 cells relative to Ad-LacZ transduced cells was quantitated by real-time PCR. (B) No alteration of ROCK protein was found in DLC1-transduced cell lines. Lane 1, 7703K/Ad-LacZ; lane 2, 7703K/Ad-DLC1; lane 3, Focus/AdLacZ; and lane 4, Focus/Ad-DLC1. Western blots were incubated with antibodies against DLC1, ROCK I, ROCK II and actin.

series of sequential steps that enable a fraction of the tumor cells to migrate from the tumor mass, survive in blood and lymphatic circulation, invade and colonize distant organs $(1,15)$. This process is comprised of interactions between the tumor cells and tissues of the host, changes in actin cytoskeleton organization, alterations of cell adhesion molecules and extracellular matrix proteins, disruption of cell-to-cell junctions and inhibition of programmed cell death $(1,16-18)$. In vitro systems for cell migration and invasion have been widely used to assess metastatic potential of cancer cells, but they may not fully reproduce the microenvironment of the metastasis target (3). Based on in vitro cell invasion observations, we undertook this study, in which we show that cells from subcutaneous tumors derived from two DLC1negative HCC cell lines disseminated to liver and lung of nude mice. We also show that this pre-metastatic step was drastically reduced in cells stably transfected with DLC1. Dissemination of human HCC cells in vivo underscores the role of DLC1 deficiency in the acquisition of invasiveness and tumor cell migration to secondary sites and the remarkable ability of the DLC1 protein to block this process. Our results are consistent with the ability of DLC1 to suppress lung metastasis of breast cancer cells (6). Of note, lung is the most frequent site for extrahepatic metastases in patients with
HCC $(19,20)$. The basis for organ tropism, one of the most critical aspects of metastatic process remains elusive (3). The absence of microscopic metastases in either liver or lung in our experiments might be due to the relatively short time allowed for metastases to form, as the mice were sacrificed before tumors reached a very large size. The dormancy of the colonizing tumor cells at both organs may be an alternative explanation (21).

The existing genomic and functional evidence implicates DLC1 in the process of metastasis. Loss of chromosome $8 p$ is associated with greater metastatic potential in various models of HCC $(22,23)$. A high frequency of LOH of markers located in the vicinity of DLC1 is common in HCC but not in dysplastic liver nodules (24), and recently LOH at 8p22 containing DLC1 was associated with worse survival of early stage HCC patients after resection and may be a useful prognostic marker for this subgroup of patients (25). DLC1 is consistently down-regulated in highly invasive HCC cell lines and metastatic subclones compared to non-metastatic ones (26). While genomic deletions have been detected in HCC cell lines and primary HCC, promoter hypermethylation and histone deacetylation, however, are the predominant mechanisms for down-regulation or silencing of DLC1 in various cancers including $\mathrm{HCC}$ (5). Loss of DLC1 expression in $7703 \mathrm{~K}$ cells is due to heavy promoter hypermethylation, while Focus cells exhibit partial methylation as well as loss of DNA copy number on 8p, where DLC1 resides $(27,28)$.

Ectopic restoration of DLC1 expression in Focus and 7703K cells decreased RhoA activity, impeded cell motility associated with a significant reduction of actin stress fibers and focal adhesion molecules and an increase in cell rounding. The RhoGAP activity of DLC1 is apparently responsible for this response in HCC cells $(29,30)$. Rho proteins are implicated in the control of actin cytoskeleton organization and focal adhesion assembly and are important components of the neoplastic process and metastasis $(31,32)$. RhoGAPs stimulate the GTPase activity of Rho proteins, converting them from the active GTP-bound form to the inactive GDP bound state (33). DLC1 is a GAP specific for RhoA and Cdc42 and was recently also shown to have GAP activity for RhoB and RhoC, thus expanding the spectrum of targets of DLC1 in tumor development $(34,35)$. Using a Rho A biosensor, it has recently been demonstrated in lung cancer cells that DLC1 decreases RhoA activity at the edge of cellular protrusions, which may contribute to the cells reduced ability to migrate, a prerequisite for DLC1's metastasis suppressor function (35). An attractive explanation linking Rho GTPases to dissemination of cancer cells has been provided in an in vitro experiment demonstrating that activation of RhoA leads to an increased frequency of cell division that may promote cell detachment and dispersal through a Rho/Rhokinase pathway (36). RhoGAP-independent mechanisms may also account for the anti-oncogenic activity of DLC1. Recently DLC1 has been found to bind to the tensin family of focal adhesion proteins $(37,38)$. DLC1-mediated suppression of the migration of human lung cancer cells requires cooperation between the Rho-GAP and tensin binding domains, even though the activities of these two domains are not interdependent (38). Also, mutation of the tensin binding site reduced its inhibitory effect on cell growth and 
migration $(37,38)$. Establishment of metastases also involves tumor cell survival and resistance to apoptosis at the sites of dissemination (39). Overexpression of DLC1 in HCC, ovarian and lung cancer cells leads to apoptosis $(5,7,40)$. In lung cancer, changes in cell morphology and the nuclear translocation of DLC1 occur prior to inhibition of cell migration and the induction of caspase-3-mediated apoptosis, suggesting that DLC1 exerts its antiproliferative function in the cytoplasm and its proapoptotic activity in the nucleus (40).

We found that expression of DLC1 resulted in the downregulation of two genes associated with metastasis, OPN and MMP-9. OPN is a secretory extracellular phosphorylated glycoprotein, produced by osteoblasts and osteoclasts that is expressed in the kidney and bone and is involved in extracellular matrix formation and calcium deposition (41). Compelling evidence has accumulated showing the importance of OPN in the process of tumorigenicity and metastasis of several cancer types (42). Neutralizing antibody to OPN reduced pulmonary metastases in nude mice (12). OPN is expressed in HCC but not in adjacent tissue and is overexpressed in the majority of primary HCCs with associated metastases as well as in cell lines derived from invasive tumors $(13,43)$. OPN expression is predictive of high grade, late stage and early recurrence of HCC as well as a shorter patient survival after orthotopic liver transplantation $(44,45)$.

Local migration and extrahepatic invasion of HCC cells also require matrix metalloproteinase activity $(46,47)$. Among the members of metalloproteinase family, the role of MMP-2 and MMP-9 has been well documented in several cancer types $(48,49)$. The level of MMP-9 mRNA expression correlates with HCC invasiveness (50,51). OPN and MMP-9 are concordantly overexpressed in primary metastatic HCC and cooperate in the process of cell invasion. Recently it was found that MMP-9 cleaves OPN to generate a 5-kDa fragment that enhanced the invasiveness of HCC cell lines (13). An OPN splice variant $(\mathrm{OPN}-\mathrm{c})$ that was more highly expressed in metastatic cells and enhanced the growth of breast cancer cells in soft agar was found to be more susceptible to MMP-9 cleavage (52).

ROCK-I and ROCK-II are RhoA effectors that are involved in actin stress fiber assembly and cell migration, but their overall levels were not altered by re-expression of DLC1 in Focus and $7703 \mathrm{~K}$ cells. The details of the mechanisms by which DLC1 influences the assembly of the actin cytoskeleton and focal adhesion remain to be determined.

These observations point to a novel role for DLC1 in addition to its effects on cell morphology and adhesion. Our observations underscore the complexity of tumor cell dissemination to distant sites and provide evidence that DLC1-mediated cytoskeleton reorganization, reduced RhoA activity, and down-regulation of OPN and MMP-9 gene expression are contributing to the inhibition of HCC dissemination. Overexpression of OPN and MMP-9 and silencing of DLC1 may provide an unfavorable prognostic marker for HCC. DLC1 gene therapy using adenoviral vector, as the most useful vehicle for gene transfer, is a realistic prospect for prevention of HCC dissemination. Also, searching for dietary constituents and pharmacological agents that up-regulate DLC1 gene expression could lead to the development of drugs that might be useful for prevention of metastasis, the most harmful event during HCC progression.

\section{Acknowledgements}

This study was supported by the Intramural Research Program of the National Cancer Institute, NIH.

\section{References}

1. Weinberg RA: Moving out: invasion and metastasis. In: The Biology of Cancer. Garland Science, Taylor \& Francis Group, LLC New York, pp587-655, 2007.

2. Clark EA, Golub TR, Lander ES and Hynes RO: Genomic analysis of metastasis reveals an essential role for RhoC. Nature 406: 532-535, 2000.

3. Nguyen DX and Massague J: Genetic determinants of cancer metastasis. Nat Rev Genet 8: 341-352, 2007.

4. Yuan BZ, Miller MJ, Keck CL, Zimonjic D, Thorgeirsson SS and Popescu NC: Cloning, characterization and chromosomal localization of a gene frequently deleted in human liver cancer (DLC-1) homogous to rat RhoGAP. Cancer Res 58: 2196-2199, 1998.

5. Durkin ME, Yuan BA, Zhou X, Zimonjic BA, Lowy DR, Thorgeirsson SS and Popescu NC: DLC-1: a Rho GTPaseactivating protein and tumor suppressor. J Cell Mol Med 11: 1185-1207, 2007.

6. Goodison S, Yuan J, Sloan D, Kim R, Li C, Popescu NC and Urquidi V: The RhoGAP protein DLC-1 functions as a metastasis suppressor in breast cancer cells. Cancer Res 65: 6042-6053, 2005.

7. Zhou X, Thorgeirsson SS and Popescu NC: Restoration of DLC-1 gene expression induces apoptosis and inhibits both cell growth and tumorigenicity in human hepatocellular carcinoma cells. Oncogene 23: 1308-1313, 2004.

8. Durkin ME, Avner MR, Huh CG, Yuan BZ, Thorgeirsson SS and Popescu NC: DLC-1, a Rho GTPase-activating protein with tumor suppressor function, is essential for embryonic development. FEBS Lett 579: 1191-1196, 2005.

9. Schneider T, Osl F, Friess T, Stockinger H and Scheuer WV: Quantification of human Alu sequences by real-time PCR: an improved method to measure therapeutic efficacy of anti-metastatic drugs in human xenotransplants. Clin Exp Metastasis 19: 571-582, 2002.

10. Lee WJ, Chen WK, Wang CJ, Lin WL and Tseng TH: Apigenin inhibits HGF-promoted invasive growth and metastasis involving blocking PI3K/Akt pathway and beta4 integrin function in MDA-MB-231 breast cancer cells. Toxicol Appl Pharmacol 226: 178-191, 2008.

11. Madsen MA, Deryugina EI, Niessen S, Cravatt BF and Quigley JP: Activity-based protein profiling implicates urokinase activation as a key step in human fibrosarcoma intravasation. J Biol Chem 281: 15997-16005, 2006.

12. Ye QH, Qin LX, Forgues M, He P, Kim JW, Peng AC, Simon R, Li Y, Robles AI, Chen Y, Ma ZC, Wu ZQ, Ye SL, Liu YK, Tang ZY and Wang XW: Predicting hepatitis B virus-positive metastatic hepatocellular carcinomas using gene expression profiling and supervised machine learning. Nat Med 9: 416-423, 2003.

13. Takafuji V, Forgues M, Unsworth E, Goldsmith P and Wang XW: An osteopontin fragment is essential for tumor cell invasion in hepatocellular carcinoma. Oncogene 26: 6361-6371, 2007.

14. Wong CL, Ching YP and Ng IO: Roles and regulations of Rhokinases in hepatocellular carcinoma. Proc Am Assoc Cancer Res 48: 318, 2007.

15. Ramaswamy S, Ross KN, Lander ES and Golub TR: A molecular signature of metastasis in primary solid tumors. Nat Genet 33: 49-54, 2003

16. Fidler IJ: Host and tumour factors in cancer metastasis. Eur J Clin Invest 20: 481-486, 1990.

17. Nicolson GL: Organ specificity of tumor metastasis: role of preferential adhesion, invasion and growth of malignant cells at specific secondary sites. Cancer Metastasis Rev 7: 143-188, 1988 .

18. Steeg PS: Tumor metastasis: mechanistic insights and clinical challenges. Nat Med 12: 895-904, 2006. 
19. Katyal S, Oliver JH III, Peterson MS, Ferris JV, Carr BS and Baron RL: Extrahepatic metastases of hepatocellular carcinoma Radiology 216: 698-703, 2000.

20. Natsuizaka M, Omura T, Akaike T, Kuwata Y, Yamazaki K, Sato T, Karino Y, Toyota J, Suga T and Asaka M: Clinical features of hepatocellular carcinoma with extrahepatic metastases. J Gastroenterol Hepatol 20: 1781-1787, 2005.

21. Goodison S, Kawai K, Hihara J, Jiang P, Yang M, Urquidi V, Hoffman RM and Tarin D: Prolonged dormancy and site-specific growth potential of cancer cells spontaneously disseminated from non-metastatic breast tumors as revealed by labeling with green fluorescent protein. Clin Cancer Res 9: 3808-3814, 2003.

22. Qin LX, Tang ZY, Sham JS, Ma ZC, Ye SL, Zhou XD, Wu ZQ and Trent JM: The association of chromosome $8 p$ deletion and tumor metastasis in human hepatocellular carcinoma. Cancer Res 59: 5662-5665, 1999.

23. Qin LX, Tang ZY, Ye SL, Liu YK, Ma ZC, Zhou XD, Wu ZQ, Lin ZY, Sun FX, Tian J, Guan XY, Pack SD and Zhuang ZP: Chromosome $8 \mathrm{p}$ deletion is associated with metastasis of human hepatocellular carcinoma when high and low metastatic models are compared. J Cancer Res Clin Oncol 127: 482-488, 2001.

24. Kahng YS, Lee YS, Kim BK, Park WS, Lee JY and Kang CS: Loss of heterozygosity of chromosome $8 \mathrm{p}$ and $11 \mathrm{p}$ in the dysplastic nodule and hepatocellular carcinoma. J Gastroenterol Hepatol 18: 430-436, 2003.

25. Pang JZ, Qin LX, Ren N, Hei ZY, Ye QH, Jia WD, Sun BS, Lin GL, Liu DY, Liu YK and Tang ZY: Loss of heterozygosity at D8S298 is a predictor for long-term survival of patients with tumor-node-metastasis stage I of hepatocellular carcinoma. Clin Cancer Res 13: 7363-7369, 2007.

26. Song LJ, Ye SL, Wang KF, Weng YQ, Liang CM, Sun RX, Zhao Y, Liu YK and Tang ZY: Relationship between DLC1 expressions and metastasis in hepatocellular carcinoma. Zhonghua Gan Zang Bing Za Zhi 13: 428-431, 2005.

27. Yuan BZ, Durkin ME and Popescu NC: Promoter hypermethylation of DLC-1, a candidate tumor suppressor gene, in several common human cancers. Cancer Genet Cytogenet 140: 113-117, 2003.

28. Zimonjic DB, Keck CL, Thorgeirsson SS and Popescu NC: Novel recurrent genetic imbalances in human hepatocellular carcinoma cell lines identified by comparative genomic hybridization. Hepatology 29: 1208-1214, 1999.

29. Wong CM, Yam JW, Ching YP, Yau TO, Leung TH, Jin DY and Ng IO: Rho GTPase-activating protein deleted in liver cancer suppresses cell proliferation and invasion in hepatocellular carcinoma. Cancer Res 65: 8861-8868, 2005.

30. Kim TY, Lee JW, Kim HP, Jong HS, Kim TY, Jung M and Bang YJ: DLC-1, a GTPase-activating protein for Rho, is associated with cell proliferation, morphology, and migration in human hepatocellular carcinoma. Biochem Biophys Res Commun 355: 72-77, 2007.

31. Jaffe $A B$ and Hall A: Rho GTPases in transformation and metastasis. Adv Cancer Res 84: 57-80, 2002.

32. Ridley AJ: Rho proteins and cancer. Breast Cancer Res Treat 84: 13-19, 2004.

33. Moon SY and Zheng Y: Rho GTPase-activating proteins in cell regulation. Trends Cell Biol 13: 13-22, 2003.

34. Wong CM, Lee JM, Ching YP, Jin DY and Ng IO: Genetic and epigenetic alterations of DLC-1 gene in hepatocellular carcinoma. Cancer Res 63: 7646-7651, 2003.

35. Healy KD, Hodgson L, Kim TY, Shutes AT, Maddileti S, Juliano RL, Hahn KM, Harden TK, Bang YJ and Der CJ: DLC1 suppresses non-small lung cancer growth and invasion by RhoGAP-dependent and independent mechanisms. Mol Carcinog DOI: 10.1002/mc.20389, 2007.
36. Vasiliev JM, Omelchenko T, Gelfand IM, Feder HH and Bonder EM: Rho overexpression leads to mitosis-associated detachment of cells from epithelial sheets: a link to the mechanism of cancer dissemination. Proc Natl Acad Sci USA 101: 12526-12530, 2004

37. Liao YC, Si L, deVere White RW and Lo SH: The phosphotyrosine-independent interaction of DLC-1 and the SH2 domain of cten regulates focal adhesion localization and growth suppression activity of DLC-1. J Cell Biol 176: 43-49, 2007.

38. Qian X, Li G, Asmussen HK, Asnaghi L, Vass WC, Braverman R, Yamada KM, Popescu NC, Papageorge AG and Lowy DR: Oncogenic inhibition by a deleted in liver cancer gene requires cooperation between tensin binding and Rho-specific GTPase-activating protein activities. Proc Natl Acad Sci USA 104: 9012-9017, 2007.

39. Mehlen P and Puisieux A: Metastasis: a question of life or death. Nat Rev Cancer 6: 449-458, 2006

40. Yuan BZ, Jefferson AM, Millecchia L, Popescu NC and Reynolds SH: Morphological changes and nuclear translocation of DLC1 tumor suppressor protein precede apoptosis in human non-small cell lung carcinoma cells. Exp Cell Res 313 3868-3880, 2007

41. Uede T, Katagiri Y, Iizuka J and Murakami M: Osteopontin, a coordinator of host defense system: a cytokine or an extracellular adhesive protein? Microbiol Immunol 41: 641-648, 1997.

42. Rittling SR and Chambers AF: Role of osteopontin in tumour progression. Br J Cancer 90: 1877-1881, 2004.

43. Luo JH, Ren B, Keryanov S, Tseng GC, Rao UN, Monga SP, Strom S, Demetris AJ, Nalesnik M, Yu YP, Ranganathan S and Michalopoulos GK: Transcriptomic and genomic analysis of human hepatocellular carcinomas and hepatoblastomas. Hepatology 44: 1012-1024, 2006.

44. Wang X: Osteopontin overexpression in hepatocellular carcinoma is a predictor of survival after liver transplantation. Hepatology 42: 391A, 2005.

45. Pan HW, Ou YH, Peng SY, Liu SH, Lai PL, Lee PH, Sheu JC, Chen CL and Hsu HC: Overexpression of osteopontin is associated with intrahepatic metastasis, early recurrence, and poorer prognosis of surgically resected hepatocellular carcinoma. Cancer 98: 119-127, 2003

46. McKenna GJ, Chen Y, Smith RM, Meneghetti A, Ong C, McMaster R, Scudamore CH and Chung SW: A role for matrix metalloproteinases and tumor host interaction in hepatocellular carcinomas. Am J Surg 183: 588-594, 2002.

47. Theret N, Musso O, Turlin B, Lotrian D, Bioulac-Sage P, Campion JP, Boudjema K and Clement B: Increased extracellular matrix remodeling is associated with tumor progression in human hepatocellular carcinomas. Hepatology 34: 82-88, 2001.

48. Van Kempen LC and Coussens LM: MMP9 potentiates pulmonary metastasis formation. Cancer Cell 2: 251-252, 2002.

49. Turpeenniemi-Hujanen T: Gelatinases (MMP-2 and -9) and their natural inhibitors as prognostic indicators in solid cancers. Biochimie 87: 287-297, 2005.

50. Ashida K, Nakatsukasa H, Higashi T, Ohguchi S, Hino N, Nouso K, Urabe Y, Yoshida K, Kinugasa N and Tsuji T: Cellular distribution of 92-kd type IV collagenase/gelatinase $\mathrm{B}$ in human hepatocellular carcinoma. Am J Pathol 149: 1803-1811, 1996.

51. Lin LI, Ke YF, Ko YC and Lin JK: Curcumin inhibits SKHep-1 hepatocellular carcinoma cell invasion in vitro and suppresses matrix metalloproteinase-9 secretion. Oncology 55: 349-353, 1998.

52. He B, Mirza M and Weber GF: An osteopontin splice variant induces anchorage independence in human breast cancer cells. Oncogene 25: 2192-2202, 2006. 